Yu Zhao ${ }^{1-3, *}$

\title{
The mysterious relation between inflammation and prostate cancer
}

DOI: $10.1515 / \mathrm{ii}-2017-0154$

Received August 23, 2017; accepted August 30, 2017; published online November 28, 2017

\begin{abstract}
Recent studies showed that inflammation is a critical cause for initiation and/or development of many cancers. In prostate cancer (PC), the inflammatory cells usually populate an immune-competent organ. This inflammatory organ can be involved in the initiation and progression of PC. Here, we mainly focus on the role of inflammation in the PC and progression of castration-resistant PC (CRPC). Moreover, we summarize the roles of inflammation factors (such as chemokines and cytokines) in PC and CRPC. Taken together, this review gives an insight into therapy for PC and CRPC through anti-inflammation.
\end{abstract}

Keywords: Inflammation, prostate cacner, CRPC, inflammation factors

\section{Introduction}

The hypothesis that inflammation causes cancer cell proliferation emerged about 150 years ago [1]. The logical relationship between cancer and inflammation is not novel today. However, recent studies have expanded the concept that inflammation is involved in cancer progression \{Karan, 2016 \#1364\}. It has been made clear that the cancer microenvironment, which is mainly populated by inflammatory cells, is an indispensable participant in the neoplastic process, metastasis, fostering of proliferation, and survival [2,3]. Additionally, cancer cells have signaling molecules similar to those of the innate immune system, such as chemokines and cytokines.

It has been accepted that inflammation cells have strong effects on cancer development $[2,4,5]$. The inflammatory cells, and the chemokines and cytokines that they produce, influence the whole tumor organ, regulating the growth, differentiation, and migration of all cells in the cancer microenvironment, including neoplastic cells, endothelial cells, and fibroblasts [6,7]. In the tumorigenic process, neoplastic cells also divert inflammatory mechanisms to support neoplastic metastasis $[2,8,9]$. This may be part of an attempt by the tumor to subvert the functions of immune cells and to support cancer progression.

The prostate is an immune-competent organ. The tissue is usually composed of a small number of inflammatory cells [10-13]. In the prostate, the T-cells are located in the fibromuscular stroma and the periglandular area [14]. There are multifactorial causes to the origin of prostatic inflammation, such as viruses, bacterial infections, hormones, dietary factors, autoimmune responses, and urine reflux $[5,10,15]$.

The persistence of these factors is able to contribute to chronic prostatic inflammation. Prostate cancer (PC) is the second common cancer among American men. The role of inflammation or infection remains unclear in different cancer sites and the prostate. Recent studies in different areas, such as epidemiology, histopathology, and molecular pathology, give emerging evidence of the possible role of prostatic inflammation as an important factor involved in PC initiation and progression [16-18]. Inflammation is involved in PC progression through several mechanisms, such as inducing cellular and genomic damage, activating cellular turnover, and creating a tissue microenvironment that induces cell replication, angiogenesis, and tissue repair $[16,19]$.

${ }^{1}$ Department of Biochemistry and Molecular Biology, Mayo Clinic College of Medicine, Rochester, MN 55905, USA;

2Department of Urology, Mayo Clinic College of Medicine, Rochester, MN 55905, USA;

${ }^{3}$ Mayo Clinic Cancer Center, Mayo Clinic College of Medicine, Rochester, MN 55905, USA;

*Correspondence: Yu Zhao, E-mail: zhao.yu@mayo.edu 
The common phenomenon of chronic inflammation in pathology samples of the prostate tissues from surgery or biopsy has provided a possible correlation between chronic inflammation and PC [20-22]. Inflammation-related inflammatory cytokines, inflammatory mediators, and growth factors were found to be able to determine an uncontrolled proliferative response in rapidly dividing cells, which were more likely to undergo mutation in PC [19,23]. Several inflammation-related genes, such as RNASEL, MSR1, and MIC1, are involved in PC. These gene-coding proteins have several functions in response to inflammation, infection, and oxidative stress. Some reports showed that their mutation may reduce the possibility of preventing carcinogenesis through these pathways [16,24]. Moreover, other studies showed that chronic inflammation can induce survival and proliferative events and posttranslational DNA modifications in the prostate tissue $[20,25]$. Repeated tissue damage and oxidative stress related to this event may produce compensatory cellular disorder of proliferation, with the risk of hyperplastic cancer cell growth or neoplastic transformation [26]. It has been reported that prostatic inflammation can generate free radicals, such as nitric oxide, which can be converted by cyclooxygenase enzymes to different eicosanoids and prostaglandins. These features have been demonstrated as regulators of PC cell proliferation [27]. Usually, glutathione transferase activity defends prostate cells against the genomic damage initiated by oxidants located at sites of inflammation [20]. Methylation of chromatin DNA produces loss of this protective activity and can be involved in the transformation from inflammation to preneoplastic lesions, e.g., high-grade prostatic intraepithelial neoplasia, and then to PC [27].

Recent clinical studies using 5-year prostate biopsies demonstrated that chronic inflammation accounts for nearly $20 \%$ PC development $[28,29]$. In addition, inflammatory atrophy was found in about $40 \%$ of PC cores in the same studies. These findings supported the hypothesis that inflammation plays an important role in prostate carcinogenesis and progression $[28,29]$.

Inflammatory factors are produced in the process of inflammation, which also play an important role in the molecular signaling pathway of PC. Here, we summarize the roles of the inflammatory factors (such as chemokines and cytokines) in PC and castration-resistant PC (CRPC).

\section{Chemokines}

Chemokines serve as factors that play important roles in inflammation and cancer. It has been reported that except immune cells, chemokine receptors are highly expressed in cancer cells compared to normal cells [28]. Additionally, recent evidence has shown that chemokines are involved in cancer cell metastasization and progression. Recent studies provide evidence that several chemokines are involved in PC, including C-X-C motif chemokine ligand 8 (CXCL8), CXCL12, and C-C ligand 2 (CCL2) [30-32]. Chemokines can facilitate tumor progression and dissemination either by shaping the cell functional profile of infiltrating lymphocytes or by promoting stromal and neoplastic cells [28].

Other studies have reported that high expression of chemokine receptors may be necessary to activate cancer progressing signals. For instance, advanced aggressive PC cells express higher levels of CCR2 compared to less-aggressive gland cells, and they also show increase in metastatic capacity compared to localized PC [33]. Furthermore, increased CXCL8 and CCL2 levels in serum have been shown to be potential prognostic markers of a highly progressive PC [34,35]. Therefore, the role of chemokines in PC is very significant.

\section{Cytokines}

Multiple inflammatory cytokines have been identified as potential activators or mediators associated with PC aggressiveness [36,37]. A group [38] working on prostate found that, baseline secretion level of interleukin (IL)-8 was associated with PC aggressiveness in primary prostate cell culture from patients subjected to radical prostatectomy. They also found that the median baseline IL-8 levels were significantly lower in cases 
with nonaggressive PC compared to those having aggressive PC. Especially, the Gleason score significantly positively correlated with the basal level of IL-8 [38]. Other groups found that IL-15, IL-17, and IL-32 regulate proliferation, migration, and invasion in PC [39-41].

One of these cytokines is macrophage inhibitory cytokine 1 (MIC-1). It is a member of the transforming growth factor beta family. It has been reported that positive regulation of MIC-1 is related to PC aggressiveness and that circulating levels of MIC-1 can predict poor prognosis in PC [42]. Moreover, IL-6 is a multifunctional cytokine that is expressed in most PC cell lines, and its expression is at higher levels in cells that do not express the androgen receptor (AR) and that show an enhanced aggressiveness potential of the tumor [43]. Aberrant IL-6 signal transducer and activator of transcription-3 (STAT3) signaling and loss of p53 occur during the progression from PC to metastatic disease [44,45].

Recent studies have provided evidence on the association between one or multiple single-nucleotide polymorphisms (SNPs) in inflammation-related pathways and PC risk and aggressiveness [36,46]. An independent group evaluated 15 SNPs in five cytokine genes (IL-1B, IL-10, tumor necrosis factor [TNF]-alfa, IL-6, and IL-8) in relation to the risk of aggressive PC, and they found an association between advanced PC and an IL-8 genotype, as well as an increased risk with combined genotypes in two other cytokines, namely, IL-1B and IL-10 [47]. Another study found that Small ubiquitin-like modifier (SUMO)-specific protease 1 (SENP1), a member of the SUMOylation protease family, plays a crucial role in the regulation of AR-dependent transcription and inflammatory hypoxia signaling pathway [48]. Another group analyzed 150 human PC specimens and found that SENP1 expression is directly correlated with PC aggressiveness [48]. In fact, higher SENP1 scores corresponded with higher Gleason scores of PC patients. In addition, silencing SENP1 level in metastatic PC cells impairs the ability to metastasize to the bone [48].

Another important cytokine associated with PC development is the macrophage migration inhibitory factor (MIF) [49]. A recent study showed that high extracellular release of MIF induces neuroendocrine differentiation in PC, which stimulates AR-independent cancer progression [50]. The relevance of this study is to link chronic prostatic inflammation through extracellular MIF release to the development of aggressive AR-independent PC and CRPC.

Recently, an important finding of inflammasomes as master regulators of inflammation has been reported. Inflammasomes are a group of multimeric proteins that are composed of Nod-like receptor (NLR) protein, an apoptosis-associated speck-like protein containing a carboxyterminal caspase recruitment domain (ASC), and procaspase-1 [8]. The assembled inflammasome complex is able to activate caspase- 1 and lead to the secretion and maturation of proinflammatory cytokines IL-1 and IL-18. Subsequently, IL-1 and IL-18 cause a wide variety of biological effects associated with infections. NLRP3 is one of the famous inflammasomes, and it senses pathogens and danger signals in response to injury or infection. Overall, various stimuli, including urine reflux, uric acid crystals, bacteria, or fungi, may lead to activation of inflammasome-mediated proinflammatory cytokines in the prostate, driving cancer progression [8].

Taken together, given the recent evidence in prostate inflammation and cancer progression, inflammation can be an important factor in PC initiation and progression. It is increasingly clear that anti-inflamm-

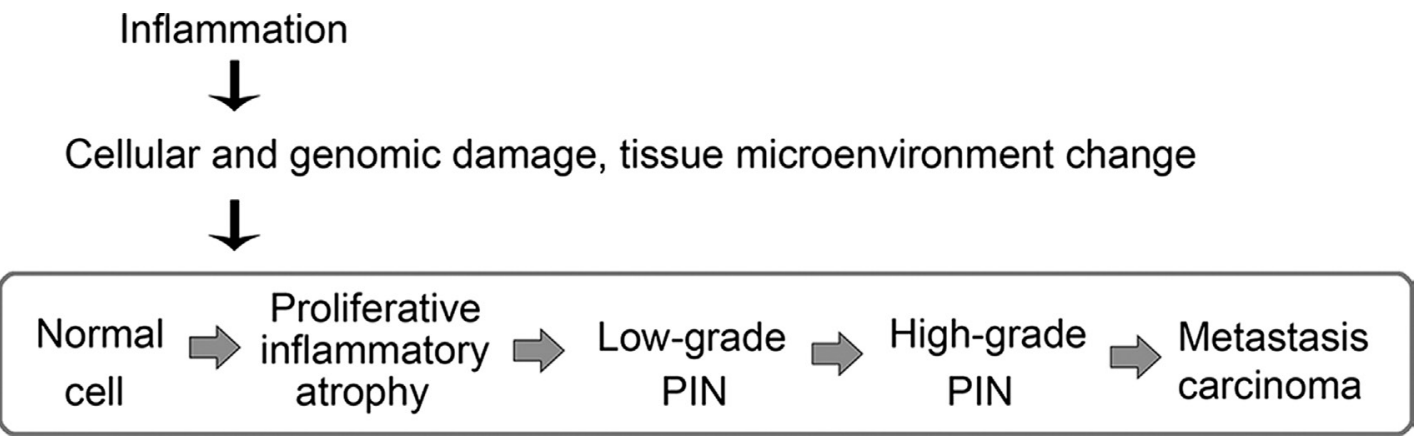

Fig. 1: Inflammation activates prostate cancer progression through cellular and DNA damage and change in tissue microenvironment. 
atory therapy will become an efficacious approach against neoplastic progression and malignant conversion (Figure 1). The elucidation of the molecular mechanism and the link between inflammation and PC will deepen our understanding of PC and expand our knowledge of PC therapy.

\section{Acknowledgments: Nil.}

Conflict of Interest: The authors declare no conflicts of interest.

\section{References}

[1] Balkwill, F., Mantovani, A. Inflammation and cancer: back to Virchow? Lancet, 2001, 357, 539-545.

[2] Coussens, L.M., Werb, Z., Inflammation and cancer, Nature, 2002, 420, 860-867.

[3] de Visser, K.E., Korets, L.V., Coussens, L.M., De novo carcinogenesis promoted by chronic inflammation is B lymphocyte dependent, Cancer Cell, 2005, 7, 411-423.

[4] Balkwill, F., Coussens, L.M., Cancer: an inflammatory link, Nature, 2004, 431, 405-406.

[5] Kruslin, B., Tomas, D., Dzombeta, T., Milkovic-Perisa, M., Ulamec, M., Inflammation in prostatic hyperplasia and carcinoma-basic scientific approach, Front. Oncol., 2017, 7, 77.

[6] Coussens, L.M., Zitvogel, L., Palucka, A.K., Neutralizing tumor-promoting chronic inflammation: a magic bullet? Science, 2013, 339, 286-291.

[7] DeNardo, D.G., Johansson, M., Coussens, L.M., Inflaming gastrointestinal oncogenic programming, Cancer Cell, 2008 , 14, 7-9.

[8] Karan, D., Dubey, S., From inflammation to prostate cancer: the role of inflammasomes, Adv. Urol., 2016, $2016,3140372$.

[9] Polackwich, A.S., Shoskes, D.A., Chronic prostatitis/chronic pelvic pain syndrome: a review of evaluation and therapy, Prostate Cancer Prostatic Dis., 2016, 19, 132-138.

[10] Gandaglia, G., Briganti, A., Gontero, P., Mondaini, N., Novara, G., Salonia, A., et al., The role of chronic prostatic inflammation in the pathogenesis and progression of benign prostatic hyperplasia (BPH), BJU Int., 2013, 112, $432-441$.

[11] Garcia-Hernandez, M.L., Uribe-Uribe, N.O., Espinosa-Gonzalez, R., Kast, W.M., Khader, S.A., Rangel-Moreno, J., A unique cellular and molecular microenvironment is present in tertiary lymphoid organs of patients with spontaneous prostate cancer regression, Front. Immunol., 2017, 8, 563.

[12] Calabria, F., Chiaravalloti, A., Ciccio, C., Gangemi, V., Gulla, D., Rocca, F., et al., PET/CT with 18F-choline: physiological whole bio-distribution in male and female subjects and diagnostic pitfalls on 1000 prostate cancer patients: $18 \mathrm{~F}$-choline PET/CT bio-distribution and pitfalls, A southern Italian experience, Nucl. Med. Biol., 2017, 51, 40-54.

[13] Sciarra, A., Gentilucci, A., Salciccia, S., Pierella, F., Del Bianco, F., Gentile, V., et al., Prognostic value of inflammation in prostate cancer progression and response to therapeutic: a critical review, J. Inflamm. (Lond), 2016, 13, 35.

[14] Bostwick, D.G., de la Roza, G., Dundore, P., Corica, F.A., Iczkowski, K.A., Intraepithelial and stromal lymphocytes in the normal human prostate, Prostate, 2003, 55, 187-193.

[15] De Marzo, A.M., Platz, E.A., Sutcliffe, S., Xu, J., Gronberg, H., Drake, C.G., et al., Inflammation in prostate carcinogenesis, Nat. Rev. Cancer, 2007, 7, 256-269.

[16] De Nunzio, C., Kramer, G., Marberger, M., Montironi, R., Nelson, W., Schroder, F., et al., The controversial relationship between benign prostatic hyperplasia and prostate cancer: the role of inflammation, Eur. Urol., 2011, 60, 106-117.

[17] Drake, C.G., Prostate cancer as a model for tumour immunotherapy, Nat. Rev. Immunol., 2010, 10, 580-593.

[18] Sfanos, K.S., Hempel, H.A., De Marzo, A.M., The role of inflammation in prostate cancer, Adv. Exp. Med. Biol., 2014, 816, 153-181.

[19] Caruso, C., Balistreri, C.R., Candore, G., Carruba, G., Colonna-Romano, G., Di Bona, D., et al., Polymorphisms of pro-inflammatory genes and prostate cancer risk: a pharmacogenomic approach, Cancer Immunol. Immunother., 2009, 58, 1919-1933.

[20] Sciarra, A., Di Silverio, F., Salciccia, S., Autran Gomez, A.M., Gentilucci, A., Gentile, V., Inflammation and chronic prostatic diseases: evidence for a link? Eur. Urol., 2007, 52, 964-972.

[21] Sciarra, A., Mariotti, G., Salciccia, S., Autran Gomez, A., Monti, S., Toscano, V., et al., Prostate growth and inflammation, J. Steroid Biochem. Mol. Biol., 2008, 108, 254-260.

[22] Cutruzzola, F., Giardina, G., Marani, M., Macone, A., Paiardini, A., Rinaldo, S., et al., Glucose metabolism in the progression of prostate cancer, Front. Physiol., 2017, 8, 97.

[23] Klein, E.A., Silverman, R., Inflammation, infection, and prostate cancer, Curr. Opin. Urol., 2008, 18, 315-319.

[24] Nelson, W.G., De Marzo, A.M., Isaacs, W.B., Prostate cancer, N. Engl. J. Med., 2003, 349, 366-381.

[25] Naber, K.G., Weidner, W., Chronic prostatitis - an infectious disease? J. Antimicrob. Chemother., 2000, 46, 157-161.

[26] Sugar, L.M., Inflammation and prostate cancer, Can. J. Urol., 2006, 13 Suppl 1, 46-47. 
[27] Palapattu, G.S., Sutcliffe, S., Bastian, P.J., Platz, E.A., De Marzo, A.M., Isaacs, W.B., et al., Prostate carcinogenesis and inflammation: emerging insights, Carcinogenesis, 2005, 26, 1170-1181.

[28] Veeranki, S., Role of inflammasomes and their regulators in prostate cancer initiation, progression and metastasis, Cell. Mol. Biol. Lett., 2013, 18, 355-367.

[29] Billis, A., Freitas, L.L., Magna, L.A., Ferreira, U., Inflammatory atrophy on prostate needle biopsies: is there topographic relationship to cancer?, Int. Braz. J. Urol., 2007, 33, 355-360; discussion 361-353.

[30] Salazar, N., Castellan, M., Shirodkar, S.S., Lokeshwar, B.L., Chemokines and chemokine receptors as promoters of prostate cancer growth and progression, Crit. Rev. Eukaryot. Gene Expr., 2013, 23, 77-91, DOI: 10.1615/CritRevEukaryotGeneExpr. 2013006905

[31] Chen, G., Liang, Y.X., Zhu, J.G., Fu, X., Chen, Y.F., Mo, R.J., et al., CC chemokine ligand 18 correlates with malignant progression of prostate cancer, Biomed. Res. Int., 2014, 2014, 230183.

[32] Maxwell, P.J., Neisen, J., Messenger, J., Waugh, D.J., Tumor-derived CXCL8 signaling augments stroma-derived CCL2-promoted proliferation and CXCL12-mediated invasion of PTEN-deficient prostate cancer cells, Oncotarget, 2014, 5, 4895-4908, DOI: 10.18632/oncotarget.2052

[33] Ellem, S.J., Taylor, R.A., Furic, L., Larsson, O., Frydenberg, M., Pook, D., et al., A pro-tumourigenic loop at the human prostate tumour interface orchestrated by oestrogen, CXCL12 and mast cell recruitment, J. Pathol., 2014, 234, 86-98.

[34] Tsaur, I., Noack, A., Makarevic, J., Oppermann, E., Waaga-Gasser, A.M., Gasser, M., et al., CCL2 chemokine as a potential biomarker for prostate cancer: a pilot study, Cancer Res. Treat., 2015, 47, 306-312.

[35] Agarwal, M., He, C., Siddiqui, J., Wei, J.T., Macoska, J.A., CCL11 (eotaxin-1): a new diagnostic serum marker for prostate cancer, Prostate, 2013, 73, 573-581.

[36] Torrealba, N., Rodriguez-Berriguete, G., Fraile, B., Olmedilla, G., Martinez-Onsurbe, P., Guil-Cid, M., et al., Expression of several cytokines in prostate cancer: correlation with clinical variables of patients. Relationship with biochemical progression of the malignance, Cytokine, 2017, 89, 105-115.

[37] Hojan, K., Kwiatkowska-Borowczyk, E., Leporowska, E., Milecki, P., Inflammation, cardiometabolic markers, and functional changes in men with prostate cancer. A randomized controlled trial of a 12-month exercise program, Pol. Arch. Intern. Med., 2017, 127, 25-35, DOI: 10.20452/pamw.3888

[38] Neveu, B., Moreel, X., Deschenes-Rompre, M.P., Bergeron, A., LaRue, H., Ayari, C., et al., IL-8 secretion in primary cultures of prostate cells is associated with prostate cancer aggressiveness, Res. Rep. Urol., 2014, 6, 27-34.

[39] Rohena-Rivera, K., Sanchez-Vazquez, M.M., Aponte-Colon, D.A., Forestier-Roman, I.S., Quintero-Aguilo, M.E., Martinez-Ferrer, M., IL-15 regulates migration, invasion, angiogenesis and genes associated with lipid metabolism and inflammation in prostate cancer, PLoS One, 2017, 12, e0172786.

[40] Zhang, Q., Liu, S., Ge, D., Cunningham, D.M., Huang, F., Ma, L., et al., Targeting Th17-IL-17 pathway in prevention of microinvasive prostate cancer in a mouse model, Prostate, 2017, 77, 888-899.

[41] Hong, J.T., Son, D.J., Lee, C.K., Yoon, D.Y., Lee, D.H., Park, M.H., Interleukin 32, inflammation and cancer, Pharmacol. Ther., 2017, 174, 127-137.

[42] Brown, D.A., Lindmark, F., Stattin, P., Balter, K., Adami, H.O., Zheng, S.L., et al., Macrophage inhibitory cytokine 1: a new prognostic marker in prostate cancer, Clin. Cancer Res., 2009, 15, 6658-6664.

[43] Culig, Z., Proinflammatory cytokine interleukin-6 in prostate carcinogenesis, Am. J. Clin. Exp. Urol., 2014, 2, $231-238$.

[44] Pencik, J., Wiebringhaus, R., Susani, M., Culig, Z., Kenner, L., IL-6/STAT3/ARF: the guardians of senescence, cancer progression and metastasis in prostate cancer, Swiss Med. Wkly., 2015, 145, w14215.

[45] Liu, G., Zhang, J., Frey, L., Gang, X., Wu, K., Liu, Q., et al., Prostate-specific IL-6 transgene autonomously induce prostate neoplasm through amplifying inflammation in the prostate and peri-prostatic adipose tissue, J. Hematol. Oncol., 2017, 10, 14.

[46] Licastro, F., Bertaccini, A., Porcellini, E., Chiappelli, M., Pernetti, R., Sanguedolce, F., et al., Alpha 1 antichymotrypsin genotype is associated with increased risk of prostate carcinoma and PSA levels, Anticancer Res., 2008, 28, 395-399.

[47] Zabaleta, J., Su, L.J., Lin, H.Y., Sierra, R.A., Hall, M.C., Sartor, A.O., et al., Cytokine genetic polymorphisms and prostate cancer aggressiveness, Carcinogenesis, 2009, 30, 1358-1362.

[48] Wang, Q., Xia, N., Li, T., Xu, Y., Zou, Y., Zuo, Y., et al., SUMO-specific protease 1 promotes prostate cancer progression and metastasis, Oncogene, 2013, 32, 2493-2498.

[49] Savoy, R.M., Ghosh, P.M., Linking inflammation and neuroendocrine differentiation: the role of macrophage migration inhibitory factor-mediated signaling in prostate cancer, Endocr. Relat. Cancer, 2013, 20, C1-C4.

[50] Tawadros, T., Alonso, F., Jichlinski, P., Clarke, N., Calandra, T., Haefliger, J.A., et al., Release of macrophage migration inhibitory factor by neuroendocrine-differentiated LNCaP cells sustains the proliferation and survival of prostate cancer cells, Endocr. Relat. Cancer, 2013, 20, 137-149. 